\title{
Die besondere Rolle der reservierten Māori-Sitze im System des personalisierten Verhältniswahlrechts Neuseelands - gelungenes Minderheitenprivileg oder überholtes Relikt kolonialen Machterhalts?
}

\author{
Von Constantin Frank-Fahle, Hamilton*
}

Vom neuseeländischen Wahlrecht sind einem internationalen Leserkreis üblicherweise drei Dinge bekannt: erstens, Neuseeland hat seit 1867 eigens eingerichtete Wahlkreise für die indigene Bevölkerung; zweitens, Neuseeland führte 1893 als erstes Land auf nationaler Ebene das aktive Frauenwahlrecht ein und drittens, die neuseeländische Bevölkerung hat sich 1993 für eine Reform des Wahlrechts zugunsten eines personalisierten Verhältniswahlsystems entschieden.

Die vorliegende Arbeit widmet sich dem ersten und dem dritten Ereignis. Es soll zunächst die geschichtliche Entstehung und Entwicklung der Māori-Wahlkreise untersucht werden ${ }^{1}$, um sodann zu analysieren, welche Rolle die Māori-Sonderwahlkreise im nunmehr geltenden System der personalisierten Verhältniswahl spielen und weshalb sie bei der Reform des neuseeländischen Wahlsystems nicht abgeschafft wurden. Abschließend wird auf den gegenwärtigen Diskurs eingegangen, um einen Ausblick auf die mögliche Entwicklung der Māori-Sitze zu geben.

\section{Siedlungshintergrund Neuseelands}

Māori sind die polynesische Bevölkerung, die als erste das neuseeländische Festland vor ca. 800 Jahren erreichte. ${ }^{2}$ Ende des 18. Jahrhunderts, als die ersten Europäer erschienen, waren die Māori eine sesshafte Landbevölkerung geworden. Zahlenangaben schwanken zwischen 100.000 und 200.000. ${ }^{3}$ Die Ankunft europäischer Siedler und die Begründung neuer Kolonien in den 1840er Jahren hatten verheerende Folgen für die demographische

Constantin Frank-Fahle, postgraduate Student an der Law School der University of Waikato, promoviert derzeit rechtsvergleichend am Lehrstuhl für Europarecht, Völkerrecht und Öffentliches Recht (Professor Dr. Markus Kotzur) an der Universität Leipzig.

Am ausführlichsten zur Wahlrechtsgeschichte der Māori: M.P.K. Sorrenson, A History of Māori Representation in Parliament, in: Royal Commission on the Electoral System (Hrsg.), Report of the Royal Commission on the Electoral System: Towards a Better Democracy, Wellington 1986, Appendix B; Neill Atkinson, Adventures in Democracy - A History of the Vote in New Zealand, Dunedin 2003.

2 Geoffrey W. Rice, The Oxford History of New Zealand, $2^{\text {nd }}$ Edition, Auckland 1992, S. 3 f.

Keith Sinclair, A History of New Zealand, Auckland 2000, S. 26. 
Entwicklung der Māori. ${ }^{4}$ Den Niedergang beschleunigten in den 1860er Jahren die militanten Konflikte zwischen Siedlern und Māori, die sogenannten Land Wars (Kriege um Land); die Zahl der Māori sank auf 70.000, schließlich, in den 1890er Jahren auf einen Tiefstand von $40.000 .^{5}$

\section{Treaty of Waitangi}

Nach der Entdeckung Neuseelands im Jahr 1642 durch den niederländischen Seefahrer Abel Tasman, kam es nach einer langen Phase der Noninterventionspolitik der britischen Krone schließlich 1840 zur Unterzeichnung des Vertrages von Waitangi durch die Krone und die verschiedenen Häupter der Māori-Stämme. ${ }^{6}$ Dieses Vertragswerk gilt als eines der zentralen Verfassungsdokumente Neuseelands und sicherte der britischen Krone die Souveränität über das neuseeländische Territorium. ${ }^{7}$ Der Vertrag regelte in drei Artikeln das Verhältnis und die gegenseitigen Verpflichtungen zwischen der Krone und den Māori. Artikel III des Vertrages garantierte den Ureinwohnern alle Rechte und Privilegien britischer Staatsbürger. ${ }^{8}$ Die Crux dieses Abkommens lag in den voneinander abweichenden Textfassungen, der englischen und der Māori-Version. ${ }^{9}$ Dass die Māori-Fassung sprachlich ungenau und wesentlich weiter gefasst war als die englische Fassung, führte in der Folgezeit zu Spannungen, die bis zu gewaltsamen Auseinandersetzungen zwischen Siedlern und Ureinwohnern eskalierten. ${ }^{10}$

Michael King, The Penguin History of New Zealand, Auckland 2003, S. 150.

5 Jack H. Nagel, Constitutional Reform and Social Difference in New Zealand, Cardozo Journal of International and Comparative Law 4 (1996), S. 378.

Morag McDowell/Duncan Webb, The New Zealand Legal System: Structures and Processes, $4^{\text {th }}$ Edition, Wellington 2006, S. $172 \mathrm{f}$.

7 Neuseeland hat im Gegensatz zu den meisten modernen Demokratien kein zentrales, schriftlich abgefasstes Verfassungsdokument. Die Verfassung ergibt sich vielmehr aus einer Reihe von Gesetzen, Statuten und Verträgen - zu denen u.a. der Treaty of Waitangi zählt. Zum Vertrag von Waitangi, seiner Entstehungsgeschichte sowie seiner völkerrechtlichen Einordnung: Ulf Tiemann, Rechte der Ureinwohner Neuseelands aus dem Vertrag von Waitangi, Münster 1999, S. 20 ff; Claudia Orange, An Illustrated History of the Treaty of Waitangi, Wellington 2004; dies., The Treaty of Waitangi, Wellington 1997.

Orginaltext Arikel III Vertrag von Waitangi: “... the Queen extends to the Natives of New Zealand Her royal protection and imparts to them all the Rights and Privileges of British Subjects".

McDowell/Webb (oben Fn. 6), S. 178; Philip A. Joseph, Constitutional \& Administrative Law in New Zealand, $3^{\text {rd }}$ Edition, Wellington 2007, S. 45 ff.

10

Peter Spiller/Jeremy Finn/Richard Boast, A New Zealand Legal History, $2^{\text {nd }}$ Edition, Wellington 2001, S. 130 f.; Raymond D. Mulholland, Introduction to the New Zealand Legal System, $10^{\text {th }}$ Edition, Wellington 2001, S. 69 f. 


\section{New Zealand Constitution Act 1852 (UK)}

Durch den New Zealand Constitution Act 1852 (UK), einen durch den imperialen Gesetzgeber erlassenen Legislativakt, wurde die neuseeländische Kolonie in sechs Provinzen, jede regiert durch einen gewählten Superintendanten und einen Provincial Council, aufgeteilt. ${ }^{11}$ Daneben trat ein nationales Repräsentantenhaus (House of Representatives), das fünfjährlich nach den Regeln des relativen Mehrheitswahlrechts (First-Past-The-Post System) in Einerwahlkreisen gewählt werden sollte. ${ }^{12}$ Auf der Grundlage der durch den Representation of the People Act 1832 (UK) im Vereinigten Königreich erreichten liberalen wahlrechtlichen Zugeständnisse basierte das aktive Wahlrecht Neuseelands auf dem Landeigentum. ${ }^{13}$ Wahlberechtigt zum Provincial Council und zum House of Representatives waren somit zunächst gemäß Section VII, VIII und XLII New Zealand Constitution Act 1852 (UK) britische Staatsangehörige, die über einen gewissen Anteil an Land verfügten. Dieses Wahlrecht bezweckte bewusst, dass sich der einzelne Bürger seine Beteiligung am politischen Willensbildungsprozess erst durch die Haltung von Grundeigentum verdienen sollte. $^{14}$

Im Hinblick auf die Māori, ergaben sich einige Hindernisse bezüglich der Teilnahme am normierten Wahlverfahren: Die Māori besaßen - im Gegensatz zur europäischen Kolonialbevölkerung - Land nicht im zivilrechtlichen Sinne europäischer Ausprägung. Ihre Stammesordnung machte es erforderlich, dass nicht der Einzelne, sondern vielmehr der jeweilige Stamm in seiner Gesamtheit das Land besaß. ${ }^{15}$ Die Māori konnten deshalb anfänglich nicht an den Wahlen zum Provincial Council und zum House of Representatives teilnehmen. Weil überdies der Prozess der Wählerregistrierung Englischkenntnisse voraussetzte, die zu Beginn der Kolonialisierungsphase den Māori fehlten, war dies ein weiteres

Zuvor wurde bereits durch den New Zealand Constitution Act 1846 (UK) eine Aufteilung Neuseelands in Provinzen vorgenommen. Hierbei waren ebenfalls Regelungen hinsichtlich der Errichtung einer repräsentativen Regierung vorgesehen. Gouverneur George Grey beantragte allerdings in der Annahme, dass die Siedler für eine eigenhändige Führung der Regierungsgeschäfte noch nicht bereit seien, eine Aufschiebung von Wahlen, so dass die Krone Wahlen zum Provincial Council, sowie zum House of Representatives bis zum Erlass des New Zealand Constitution Act (UK) 1852 ausgesetzt hatte. Zum Ganzen: McDowell/Webb, (oben Fn. 6), S. 91.

Wenngleich zu anfangs in den urbanen Zentren Mehrmannwahlkreise eingerichtet wurden. John E. Martin, The House: New Zealand's House of Representatives 1854-2004, Palmerston North 2004, S. 11.

Atkinson, (oben Fn. 1), S. 14.

14 Andrew Geddis, Electoral Law in New Zealand, Wellington 2007, S. 64; Keith Jackson/Alan McRobie, New Zealand Adopts Proportional Representation: Accident? Design? Evolution?, Brookefield (Vermont) 1998, S. 21. 
Hindernis in der Ausübung des Wahlrechts. ${ }^{16}$ Schließlich waren die Wahlkreise zu den ersten Wahlen so eingeteilt, dass große Teile des Landesinneren der Nord Insel - einem der Haupt-Siedlungsgebiete der Māori - nicht abgedeckt waren, da sie bisher noch nicht von Europäern besiedelt waren. ${ }^{17}$

Ursprünglich war von dem Colonial Office die Auffassung vertreten worden, dass die Māori langfristig - ebenso wie die europäischen Siedler - ihr Wahlrecht in Folge der Erfüllung der Grundeigentumserfordernisse würden ausüben können, nachdem sie nach und nach die europäische Form der Haltung von Grundeigentum übernehmen würden. ${ }^{18}$ Diese auf Assimilation fußende Betrachtung verkannte indes die Verankerung der stammesmäßigen Landhaltung innerhalb der gesellschaftlichen Struktur der Māori, die gerade eine individualisierte Stellung des Einzelnen im Gefüge des Stammes vermied, so dass selbst in den 1860er Jahren nur vergleichsweise wenige Māori an den Wahlen zum House of Representatives teilnahmen. ${ }^{19}$ Erste Versuche, der Situation Rechnung zu tragen, dass die Māori faktisch keine Rolle im politischen Leben Neuseelands spielten, wurden durch den Māori Electoral Bill 1865, den Native Commission Act 1865 sowie den Native Rights Act 1865 unternommen. ${ }^{20}$ Diese Regelungen verbesserten diesen Zustand kaum; das Problem blieb weiterhin ungelöst.

Die massiven Landerwerbungen durch die europäischen Siedler, verbunden mit der raschen Expansion neuer Siedlungsanlangen, verstärkten die bereits erwähnten Spannungen zwischen den Māori und Siedlern. Die Landkriege und die Māori-Königbewegung ${ }^{21}$ mit dem Bestreben nach Bewahrung der Kräfte und Stammesgewohnheiten stellen in diesem Zusammenhang nur die zentralsten Ereignisse dar, in denen sich die Konflikte entluden. ${ }^{22}$

Raewyn Dalziel, Towards Representative Democracy: 100 years of the Modern Electoral System, in: Atholl Anderson/David Green (Hrsg.), Towards 1990 - Seven leading Historians examine significant aspects of New Zealand History, Wellington 1989, S. 51; Anne Sullivan, Effecting Change through Electoral Politics: Cultural Identity and the Māori Franchise, The Journal of the Polynesian Society 112 (2003), S. 222.

W.K. Jackson/G.A. Wood, The New Zealand Parliament and Māori Representation, Historical Studies 11 (1964), S. 384; D.G. Herron, The Franchise and New Zealand Politics, 1853-8, Political Science 12 (1960), S. 29.

Geddis, A Dual Track Democracy? The Symbolic Role of the Māori Seats in New Zealand's Electoral System, Election Law Journal 5 (2006), S. 352.

Neill Atkinson, Parliament and the People: Towards Universal Male Suffrage in the $19^{\text {th }}$ Century New Zealand, New Zealand Journal of Public and International Law 3 (2005), S. 167.

20

Sarah A. McClelland, Māori Electoral Representation: Challenge to Orthodoxy, New Zealand Universities Law Review 17 (1997), S. 277 ff.; Brian Ritchie/Hugo Hoffmann, The Electoral Law of New Zealand - A Brief History, in: Royal Commission on the Electoral System (Hrsg.), Report of the Royal Commission on the Electoral System - Towards a Better Democracy, Wellington 1986, Appendix A, S. 29.

21 Joan Metge, The Māoris of New Zealand, London, Boston 1976, S. 196 f.; Rice, (oben Fn. 2), S. 153.

22

Sorrenson, (oben Fn. 1), S.. 15 f.; King, (oben Fn. 4), S. 210 ff. 


\section{Māori Representation Act 1867}

Diese Vorgänge gaben Anlass, die Einbeziehung der Māori in das Wahlrecht Neuseelands erneut zu überdenken und langfristige Lösungsformen zu finden, um den bisherigen Interessenkonflikten zwischen Māori und Siedlern Rechnung zu tragen, zugleich aber auch die koloniale Macht erhalten zu können. ${ }^{23}$ Ein vom Grundeigentum losgelöstes Wahlrecht war zunächst insoweit undenkbar, als dass die durch den Great Reform Act 1832 errungenen Rechte als liberal galten und das Recht zur Beteiligung am politischen Willensbildungsprozess auf die Wohlhandenden der Gesellschaft beschränkt bleiben sollte.

Durch den Māori Representation Act 1867, der 1872 auslaufen sollte, wurden den Māori sodann vier Sitze im House of Representatives eingeräumt. ${ }^{24}$ In Übereinstimmung mit Section 4 wurde das Staatsgebiet Neuseelands in vier Māori-Wahlkreise, drei auf der Nord- und einen auf der Südinsel, aufgeteilt.

Zwar symbolisiert diese Bereitstellung garantierter Sitze zweifelsohne ein Zugeständnis der europäischen Siedler an die Māori; aber dies sollte nicht überbewertet werden. Im Lichte der Bevölkerungsproportionen spiegelten die Sitze nicht den Stand der eigentlich verdienten Dichte an Repräsentation wider: Die Māori hatten vier Sitze, um eine geschätzte Bevölkerung von 40.000 bis 50.000 zu repräsentieren, während den europäischen Siedlern 72 Repräsentanten für 220.000 Menschen zustanden. Bei einer verhältnismäßigen Sitzverteilung hätten den Māori zwischen 14 und 16 Sitze im Parlament zugestanden. ${ }^{25}$ In der Folgezeit stellten die vier Sitze zumindest zeitweise - insbesondere am Ende des 19. und zu Beginn des 20. Jahrhunderts - insoweit eine proportionale Zuteilung dar, als dass die „europäischen“ Parlamentssitze verringert wurden. ${ }^{26}$ Weiterhin ergab sich aus dem Umstand der Fixierung der Sitze auf eine Anzahl von vier, dass die Größe der Wahlkreise einerseits einen regelmäßigen Kontakt zwischen Wählern und Wahlkreisabgeordneten faktisch ausschlossen und andererseits durch die zu überwindenden Distanzen die Wahlkampfkosten der Māori-Abgeordneten in die Höhe getrieben wurden. ${ }^{27}$ Kurz vor ihrem Auslaufen verlängerte das Parlament diese Regelung um weitere vier Jahre, was die MāoriSitze im Jahre $1876 \mathrm{zu}$ einem festen Bestandteil des neuseeländischen Wahlsystems machte. $^{28}$

Die Erforderlichkeit der Errichtung gesonderter Wahlkreise für Māori ergab sich damit in der Gesamtheit aus folgenden Gründen: erstens, aus der Notwendigkeit, ein bezwunge-

Augie Fleras, From Social Control towards Political Self-Determination? Māori Seats and the Politics of Separate Māori Representation in New Zealand, Canadian Journal of Political Science 18 (1985), S. 555.

Section 3 Māori Representation Act 1867.

Sorrenson, (oben Fn. 1), S. 21.

26

27

Nagel, (oben Fn. 5), S. 383.

Metge, (oben Fn. 21), S. 192.

28

Geddis, (oben Fn. 14), S. 97. 
nes Volk, das zum Aufbau der Kolonie unentbehrlich war, in den politischen Willensbildungsprozess zu integrieren; zweitens, so schnell wie möglich eine Assimilation herbeizuführen, ohne dabei der jungen Kolonie eine Belastung aufzuerlegen; drittens, die Interessen der Siedler abzusichern, bis die Landübernahme soweit fortgeschritten war, dass die Landkäufe konsolidiert werden konnten, sowie viertens, Versuchen zur Etablierung einer separaten Repräsentationsform durch Māori zuvorzukommen, die die parlamentarische Souveränität des House of Representatives möglicherweise hätten unterwandern können. ${ }^{29}$

\section{Electoral Act 1956}

Der Electoral Act 1956 brachte neben der Konsolidierung aller bisherigen Reformierungen des Wahlrechts als Neuerung hervor, dass wesentliche Aspekte des Wahlrechts, wie etwa das Wahlsystem und die Besetzung der Representation Commission, einer erschwerten legislativen Abänderbarkeit unterworfen wurden. So konnte das Wahlsystem der relativen Mehrheitswahl nunmehr nur durch 3/4 Mehrheit im House of Representatives oder durch Referendum abgeändert werden. ${ }^{30}$ Die Māori-Sitze ihrerseits waren von diesem „Single Entrenchment ${ }^{* 31}$ ausgenommen und somit nicht abgesichert.

\section{Electoral Amendment Act 1975}

Das Prinzip der strikt dualen Repräsentation wurde erstmals 1975 durch den Electoral Amendment Act durchbrochen, indem den Māori ein Optionsrecht (Māori Electoral Option) zugestanden wurde, zu wählen, ob sie ihr Wahlrecht in den Māori oder den generellen Wahlkreisen ausüben wollten. ${ }^{32}$ Diese Lockerung der strengen Trennung von Māori und europäischen ${ }^{33}$ Wählern auf Wahlkreisebene ist Ergebnis eines Emanzipierungsprozes-

29

30

31

Das Modell des „Single Entrenchment“ wurde im Hinblick auf das auf der Westminster Tradition beruhenden Prinzip der Parlamentssouveränität (näher hierzu: Christoph von Bernstorff, Einführung in das englische Recht, 3. Auflage, München 2006, S. 42 ff.) problematisiert. Da die Regelung, die eine qualifizierte Mehrheit zur Abänderbarkeit verlangt, ihrerseits indes nicht abgesichert ist, kann ein nachfolgendes Parlament die Absicherung durch die Aufhebung der absichernden Regelung erreichen, um in einen nächsten Schritt die vormals abgesicherten Wahlrechtsregelungen aufzuheben oder abzuändern. Insoweit ist der Streit lediglich dogmatischer Natur. Anders wäre die Lage indes bei einem sogenannten „Double Entrenchment“, also bei einer Absicherung der Regelung, die gerade eine qualifizierte Mehrheit zur Absicherung fordert. Eine derartige Regelung wäre wohl mit dem Prinzip der Parlamentssouveränität nicht mehr vereinbar. Zum Ganzen: Joseph, (oben Fn 9), S. 123/124, 487/488; McDowell/Webb, (oben Fn. 6), S. 111; Mulholland, (oben Fn. 10), S. 28; K.J. Scott, The New Zealand Constitution, Oxford 1962, S. 7 f.
Unter dem Electoral Act 1956 war es lediglich Halbabkömmlingen gestattet zu wählen, ob sie ihr Wahlrecht im generellen oder Māori-Wahlkreis ausüben möchten.

Die Terminologie ist insoweit ungenau, als dass sie verkennt, dass Asiaten und Pazifikinsulaner traditionellerweise ebenfalls im Rahmen der generellen Wahlkreise ihr Wahlrecht ausüben. 
ses, der maßgeblich durch die Land March Bewegung initiiert wurde: ${ }^{34}$ Die Land March Bewegung im Jahr 1975 bestand aus einem Protestmarsch vor das nationale Parlament in Wellington. Die Bewegung richtete sich gegen die Negierung der Māori-Rechte aus dem Vertrag von Waitangi. ${ }^{35}$ Aufgrund dieses politischen Drucks wurde u.a. das Waitangi Tribunal, eine Art Schiedsgericht, das die Erfüllung der Obligationen aus dem Vertrag von Waitangi untersuchen sollte, errichtet. ${ }^{36}$

Zwischenzeitliche Reformversuche, die Māori-Sitze abzuschaffen, blieben erfolglos. Reformbestrebungen dahingehend, dass die Zahl flexibel gestaltet werden sollten, also anhand der für die Māori-Wahlkreise registrierten Wähler proportional zu den generellen Wahlkreisen ausgestaltet werden sollten, blieben ebenso ohne Erfolg. Diese Idee war ursprünglich von der amtierenden Labour Regierung in die Debatte gebracht worden, wurde indes sogleich nach ihrer Ablösung 1975 verworfen. ${ }^{37}$

\section{Reformierung des Wahlrechts}

Neuerliche Reformbestrebungen hinsichtlich der Māori-Wahlkreise setzten erst wieder ein, als das relative Mehrheitswahlrecht als taugliches Wahlsystem generell in Frage gestellt wurde. Der Reformprozess gab in der vergleichenden Politikwissenschaft insoweit Grund zu näherer Untersuchung, als dass bisher angenommen wurde, dass ein fundamentaler Wechsel eines Wahlsystems - etwa vom Mehrheits- zum Verhältniswahlsystem oder umgekehrt - notwendigerweise mit einem konstitutionellen Moment, also der generellen Infragestellung des staatsorganisatorischen Aufbaus einhergehen müsse. ${ }^{38}$ In diesem Rahmen wird lediglich auf die Reformimpulse im Hinblick auf die Māori-Sitze unter Benennung der maßgeblichen reformimitierenden Kriterien für die Wahlsystemreform eingegangen werden. ${ }^{39}$ Aus dem komplexen Ursachengefüge des Reformprozesses soll daher auszugsweise

34

35

36

37

38

39

Rice, (oben Fn. 2), S. 573.

Orange, The Treaty of Waitangi, (oben Fn. 7), S. 248.

Sections 4 und 5 Treaty of Waitangi Act 1975; Amy L. Catalinac, The Establishment and Subsequent Expansion of the Waitangi Tribunal: The Politics of Agenda Setting, Political Science 56 (2004), S. 5-22.

Sorrenson, (oben Fn. 1), S. 52/53; Elisabeth M. McLeay, Political Argument about Representation: The Case of the Māori Seats, Political Studies 28 (1980), S. 48.

Dieter Nohlen, Changes and Choices in Electoral Systems, in: Arend Lijphart/Bernhard Grofman (Hrsg.), Choosing an Electoral System, New York 1984, S. 218 f.

Deutschsprachige Untersuchungen haben den Reformprozess näher beschrieben: Steffanie Richter, Modell Aotearoa - Der Prozeß der Wahlsystemreform in Neuseeland, Berlin, Cambridge 1999 sowie Caroline Brummet, Die Reform des neuseeländischen Wahlsystems - Ursachen, Prozeß, Auswirkungen, Frankfurt am Main, Berlin, Bern, Brüssel, New York, Oxford, Wien 2000; Dieter Nohlen, Wahlrecht und Parteiensystem, 5. Auflage, Opladen, Farmington Hills 2007, S. 208 ff. Aus den kaum mehr zu überschaubaren englischsprachigen Publikationen sollen hier nur folgende Arbeiten genannt werden: Jackson/McRobie, (oben Fn. 14); David Denemark, Choosing MMP in New Zealand: Explaining the 1993 Electoral Reform, in: Matthew S. Shugart/Martin P. Watten- 
der Umstand genannt werden, dass die Wahlen in den Jahren 1978 und 1981 den Hauptnachteil des Mehrheitswahlsystems spiegelten, indem nämlich der gesamtstimmenmäßige Verlierer, in diesem Fall die National Party, in der Lage war, die Regierung zu stellen. ${ }^{40}$ Auf dieser Grundlage bildete die Labour Partei, die von 1984 bis 1990 die Regierung stellte, 1985 eine "Royal Commission on the Electoral System". ${ }^{4}$

\subsection{Royal Commission on the Electoral System 1985-1986}

Die eingerichtete Royal Commission on the Electoral System untersuchte verschiedene Wahlsysteme auf ihre Tauglichkeit für Neuseeland. Die Kommission sollte dabei der gewachsenen legislativen Anerkennung von Rechten der Ureinwohner besondere Aufmerksamkeit schenken, indem sie die Wahlsysteme dabei u.a. auf das Kriterium der effektiven Repräsentation der Māori untersuchen sollte. ${ }^{42}$ Unter den fünf Mitgliedern der Kommission befand sich u.a. Whetumaramara Wereta, eine Māori, die insbesondere die Bewahrung von Māori-Interessen überwachen sollte. ${ }^{43}$

\subsection{Empfehlungen der Royal Commission on the Electoral System}

Nach umfassender Prüfung verschiedener Wahlsysteme sprach sich die Royal Commission 1986 dafür aus, dass die neuseeländische Bevölkerung im Rahmen eines Referendums über die Ablösung des Mehrheitswahlrechts durch ein Verhältniswahlrecht nach deutscher Ausprägung entscheiden sollte. ${ }^{44}$ Darüberhinaus riet sie dazu, im Falle der Übernahme eines Systems der personalisierten Verhältniswahl (Mixed-Member-Proportional System ${ }^{45}$ ), die reservierten Māori-Sitze abzuschaffen. ${ }^{46}$ Begründet wurde diese Empfehlung im wesentlichen mit der Annahme, dass das personalisierte Verhältniswahlsystem genügend Möglich-

berg (Hrsg.), Mixed-Member Electoral Systems - The Best of Both Worlds?, Oxford 2001, S. 7095.

40

Jack Vowles, The Politics of Electoral Reform in New Zealand, International Political Science Review 16 (1995), S. 100; Stephen Ingle, Electoral Reform in New Zealand: The Implications for Westminster Systems, The Journal of Legislative Studies 1 (1995), S. 78.

41

42

43

44

45

46

Jack Vowles, New Zealand: The Consolidation of Reform?, in: Michael Gallagher/Paul Mitchell (Hrsg.), The Politics of Electoral Systems, Oxford 2005, S. 296.

Royal Commission on the Electoral System, Report of the Royal Commission on the Electoral System, Wellington 1986, S. 9.

Denemark, (oben Fn. 39), S. 85.

Royal Commission on the Electoral System, (oben Fn. 42), S. 64/65.

Zur Terminologie Mixed-Member-Proportional System: Matthew S. Shugart/Martin P. Wattenberg, Mixed-Member Electoral Systems: A Definition and Typology, in: Matthew S. Shugart/ Martin P. Wattenberg (Hrsg.), Mixed-Member Electoral Systems - The Best of Both Worlds?, Oxford 2001, S. 9-24; Federico Ferrara/Erik S. Herron/Misa Nishikawa, Mixed Electoral Systems - Contamination and its Consequences, New York 2005, S. 16 ff.

Royal Commission on the Electoral System, (oben Fn. 42), S. 108. 
keiten biete eine hinreichende Repräsentation der Māori zu gewährleisten. ${ }^{47}$ Insbesondere sei durch die proportionale Verteilung der Parlamentssitze die Gelegenheit eröffnet, durch Formung von Māori Parteien, diese anteilmäßig an der Sitzverteilung zu berücksichtigen. Sollte die Gründung neuer Māori Parteien ausbleiben, würden Māori-Interessen jedenfalls in die Agenda der bestehenden Parteien aufgenommen, da diese nunmehr ebenfalls, in Anerkennung der demographischen Stärke, um das nicht unerhebliche Stimmgewicht der Māori ${ }^{48}$ kämpfen müssten. ${ }^{49}$ Schließlich befürwortete die Kommission für den Fall der Abschaffung der Sitze eine Ausnahmeregelung von der $5 \%$-Hürdenbestimmung nach deutschem Vorbild $^{50}$ für Parteien, die überwiegend Māori oder Māori-Interessen repräsentieren. $^{51}$

\subsection{Parlamentarische Beratung der Vorschläge der Royal Commission}

Eine parlamentarische Untersuchungskommission (Electoral Law Committee) setzte sich im Anschluss mit den von der Royal Commission vorgegeben Kriterien zur Reformierung des Wahlsystems auseinander. 1988 wurde zunächst festgestellt, dass das bisherige Mehrheitswahlsystem beibehalten werden sollte. ${ }^{52}$ In der Folgezeit wurde der Ruf nach einer grundsätzlichen Reform indes insbesondere unter dem Gesichtspunkt laut, dass die Royal Commission überzeugend und unzweideutig dargelegt hatte, dass das geltende Mehrheitswahlsystem nicht zu einer gerechten Repräsentation führe. ${ }^{53}$ Dementsprechend nahm die National Party 1989/1990 in ihr Wahlkampfprogramm auf, ein indikatives Referendum über die Frage einer Wahlsystemreform zu halten. ${ }^{54}$

Mittlerweile besteht die neuseeländische Bevölkerung aus 4.027.947 Bürgern, von denen 565.329, also $14 \%$, Māori Abstammung haben. Hierzu: Statistics New Zealand, Demographic Trends: 2007, Wellington 2008, S. 16.

Royal Commission on the Electoral System, (oben Fn. 42), S. 102.

Gemäß $\S 6$ Abs. 6 S. 2 Bundeswahlgesetz findet die $5 \%$-Hürdenregelung des $\S 6$ Abs. 6 S. 1 keine Anwendung, soweit es sich um eine Partei nationaler Minderheiten handelt.

Royal Commission on the Electoral System, (oben Fn. 42), S. 66.

52 Electoral Law Committee, Report of the Electoral Law Committee: Inquiry into the Report of the Royal Commission on the Electoral System, in: Jonathan Boston/Stephen Levine/Elizabeth McLeay/Nigel S. Roberts (Hrsg.), Electoral and Constitutional Change in New Zealand: An MMP Source Book, Palmerston North 1999, S. 32. Zealand, Political Science and Politics 27 (1994), S. 526.

Die Bekanntgabe des Wahlversprechens beruhte auf einem Irrtum. Zum Ganzen: Alan Renwick, Why Did National Promise a Referendum on Electoral Reform in 1990, Political Science 59 (2007), S. $8 \mathrm{f}$. 


\subsection{Referenden und Gesetzesentwürfe}

1992 wurde ein indikatives Referendum abgehalten, in dem sich die neuseeländische Bevölkerung mit einer Mehrheit von 84,7 \% für eine Reform des Wahlsystems aussprach. ${ }^{55}$ Daraufhin wurde eine parlamentarische Kommission (Electoral Law Select Committee) mit einem Gesetzesentwurf für ein neues Wahlrecht beauftragt. ${ }^{56}$ Die Kommission beriet u.a. über die Rolle der Māori-Sitze im Hinblick auf eine bevorstehende Übernahme des personalisierten Verhältniswahlsystems. In diesem Rahmen verdichtete sich die Handhabung der Māori-Sitze auf vier Optionen: erstens, in Übereinstimmung mit der Empfehlung der Royal Commission, die Māori-Sitze abzuschaffen, zweitens, die Māori-Sitze in ihrer bisherigen Form beizubehalten und zusätzliche vier Sitze zu den angestrebten $120^{57}$ Parlamentssitzen zu gewähren, drittens, vier Māori-Sitze abzüglich der 120 Parlamentssitze bereitzustellen, sowie viertens, die Māori-Sitze variabel auf Grundlage der tatsächlich für die Māori-Wahlkreise eingetragenen Wähler auszugestalten. ${ }^{58}$

In der Folge formierte sich eine Opposition im Hinblick auf die Abschaffung der Māori-Wahlkreise. Vielfach wurde eine Weigerung der Aufhebung der Sitze mit der besonderen Stellung der Māori innerhalb der pluralistischen Gesellschaft Neuseelands begründet. $^{59}$ Diese ablehnende Auffassung war insbesondere unter den Māori verbreitet. ${ }^{60}$ Die oppositionelle Haltung der Māori ergab sich im Ergebnis insbesondere aus der Befürchtung eine garantierte Form der Repräsentation gegen eine ungewisse einzutauschen. ${ }^{61}$ Im Gegensatz dazu brachte Geoffrey Palmer, dem maßgeblichen Initiator der Wahlsystemreform, seine Kritik, auf der Grundlage der Empfehlungen der Royal Commission, an der Aufrechterhaltung der separaten Wahlkreise zum Ausdruck. ${ }^{62}$ In Ermangelung eines politischen Konsenses über die Māori-Sitze einigte man sich schließlich auf die vierte Option, die

Das indikative Referendum wurde zweistufig abgehalten: zunächst wurden die Wähler danach gefragt, ob sie das geltende Wahlsystem beibehalten wollen oder durch ein anderes ersetzen wollen. $15,3 \%$ sprachen sich für die Beibehaltung des Mehrheitswahlsystems (FPP) aus, während eine überwältigende Mehrheit von $84,7 \%$ eine Änderung wollte. In einem zweiten Schritt konnten, diejenigen, die sich für eine Änderung ausgesprochen hatten sodann zwischen mehreren Wahlsystemen wählen. Im Ergebnis stimmten in diesem Schritt 70,5\% für die Einführung eines personalisierten Verhältniswahlsystems (MMP).

Philip Temple, Changing the Rules in New Zealand: The Electoral Reform Referenda of 1992 and 1993, The Political Quarterly 66 (1995), S. 235.

Die Royal Commission hatte sich in ihren Empfehlungen u.a. dafür ausgesprochen, dass im Falle des Übergangs zur proportionalen Repräsentation das Parlament auf 120 Sitze erhöht werden sollte. Royal Commission on the Electoral System, (oben Fn. 42), S. 129.

Jackson/McRobie, (oben Fn. 14), S. 225.

Geddis, (oben Fn. 18), S. 358.

60

Atkinson, (oben Fn. 1), S. 215/216.

61

62

Geddis, (oben Fn. 18), S. 358.

Jackson/McRobie, (oben Fn. 14), S. 227. 
Māori-Sitze im Falle eines erfolgreichen bindenden Referendums, zunächst beizubehalten. Da die Fixierung der Sitze auf eine Anzahl von vier als unsachgemäß betrachtet wurde, sollte die Zahl der Sitze variabel gestaltet und auf der Grundlage der für die Māori-Wahlkreise registrierten Wähler berechnet werden, wenngleich einzelne Māori-Vertreter damit die Befürchtung verbunden, dass die Sitze möglicherweise auf einen Stand von unter vier sinken könnten. 63

1993 folgte dann neben der Wahl zum House of Representatives das bindende Referendum. Die neuseeländische Bevölkerung stimmte mit 53,9\% für einen Wechsel des Wahlsystems zugunsten des Systems einer personalisierten Verhältniswahl. ${ }^{64}$

\section{Electoral Act 1993}

Durch den Electoral Act 1993 hat Neuseeland ein Wahlsystem übernommen, das in weiten Teilen dem Wahlsystem zum deutschen Bundestag entspricht. ${ }^{65}$ Es besteht aus einer Kombination von Mehrheits- und Verhältniswahlsystem. Die Wähler haben zwei Stimmen: die Erststimme besteht zur Auswahl einer Partei und dient der Ermittlung der proportionalen Zusammensetzung des House of Representatives über eine nationale Parteiliste, während die Zweitstimme der Bestimmung eines Wahlkreisabgeordneten dient. ${ }^{66}$ Die Berechnung der proportionalen Sitzzuteilung erfolgt nach dem Sainte-Laguë-Verfahren. ${ }^{67} \mathrm{Um}$ dem Prozess einer Parteienzersplitterung entgegenzuwirken hat Neuseeland weiterhin eine $5 \%$ Hürdenregelung eingerichtet, so dass Parteien an der proportionalen Sitzverteilung lediglich teilnehmen, wenn sie mindestens $5 \%$ der Erststimmen auf sich vereinigen konnten. ${ }^{68}$ Ferner wurde durch den Electoral Act 1993 eine Ausnahmebestimmung dieser Hürdenregelung für den Fall getroffen, dass eine Partei mindestens in einem Wahlkreis ein Direktmandat erzielt. ${ }^{69}$ Eine Ausnahmeregelung dahingehend, dass Parteien nationaler Minderheiten von dieser $5 \%$-Hürdenbestimmung ausgenommen werden, hat Neuseeland, entgegen der Empfehlung der Royal Commission, hingegen nicht in den Electoral 1993 inkorporiert. Die Zusammensetzung des 120 Mitglieder umfassenden Parlaments wird alle drei Jahre bestimmt. ${ }^{70}$ Schließlich besteht die Möglichkeit der Entstehung von Überhangmandaten. ${ }^{71}$

63

64

65

66

67

68

69

70

71

Vowles, (oben Fn. 40), S. 107.

Temple, (oben Fn. 56), S. 237.

Mulholland, (oben Fn. 10), S. 63; Joseph, (oben Fn. 9, S. 343.

Section 150(3) Electoral Act 1993.

Section 191(5) Electoral Act 1993.

Section 191(4)a Electoral Act 1993.

Section 191(4)b Electoral Act 1993.

Section 17(1) New Zealand Constitution Act 1986 und Section 191(7) Electoral Act 1993.

Section 192(5) Electoral Act 1993. 
Die Māori-Sitze sind, entgegen der Empfehlung der Royal Commission on the Electoral System, weiterhin gemäß Section 45 Electoral Act 1993 Bestandteil des neuseeländischen Wahlsystems. ${ }^{72}$ Die wesentlichste Neuerung im Hinblick auf die Māori-Sitze besteht in der variablen Zahl der Sitze. Erstmals seit 1867 konnte damit die Zahl der Māori-Wahlkreise und Sitze entsprechend der tatsächlich für diese Wahlkreise registrierten Wähler anwachsen bzw. abfallen. Damit war das wahlrechtliche Gebot der Gleichheit der Wahl (,one man, one vote, one value') schließlich auch für die indigene Bevölkerung Neuseelands erreicht worden.

\section{Māori Electoral Option}

Die Anzahl der Māori-Wahlkreise hängt damit nunmehr maßgeblich davon ab, wie viele Māori ihr Wahlrecht in den Māori-Wahlkreisen ausüben möchten, mithin von ihrem Optionsrecht zugunsten der Māori-Sitze Gebrauch machen. ${ }^{73}$ Die Māori Electoral Option (MEO) ist ein Prozess der im Anschluss an die fünfjährlich ${ }^{74}$ gehaltene Volkszählung durchgeführt wird. ${ }^{75}$ Die Volkszählung dient in wahlsystematischer Perspektive der Ermittlung der Bevölkerungsdaten Neuseelands, um die Anzahl der Wahlbevölkerung im Allgemeinen und die Anzahl der Māori-Wahlbevölkerung im Besonderen zu bestimmen. ${ }^{76}$ Damit wird innerhalb der Volkszählung gesondert festgestellt wer Māori bzw. Māori-Abkömmling ist. ${ }^{77}$ Nachdem ermittelt wurde, wie viele Neuseeländer Māori sind oder sich als Māori-Abkömmling einordnen, werden denjenigen, die sich als Māori oder Māori-Abkömmlinge klassifizieren, in einer viermonatigen Phase, die sich unmittelbar an die Volkszählung anschließt, die Möglichkeit eröffnet, sich entsprechend dem Optionsrecht für die Māori oder die generellen Wahlkreise registrieren zu lassen. ${ }^{78}$

Die Berechnung der Anzahl der Māori-Wahlkreise erfolgt sodann zunächst durch Ermittlung der Māori-Wahlbevölkerung. ${ }^{79}$ Diese wird durch eine Multiplikation der MāoriBevölkerung mit dem Quotienten bestehend aus unter den Māori-Wahlkreisen registrierten Māori geteilt durch die Summe bestehend aus unter den generellen Wahlkreisen registrier-

72

74

75

76

77

78

79

Joseph, (oben Fn. 9), S. 345.

Jonathan Boston/Stephen Levine/Elizabeth McLeay/Nigel S. Roberts (Hrsg.), Electoral and Constitutional Change in New Zealand: An MMP Source Book, Palmerston North 1999, S. 101 ff. Section 23(1) Statistics Act 1975.

Der Prozess der Māori Electoral Option ist detailiert in Section 76-79 Electoral Act 1993 geregelt. Geddis, (oben Fn. 14), S. $100 \mathrm{f}$.

Die Wahlbevölkerung berechnet sich gemäß Section 3(1) Electoral Act 1993 nicht aus der Summe aller Wahlberechtigten, sondern aus der Gesamtheit aller Einwohner Neuseelands, also unter Einschluss der vom Wahlrecht ausgeschlossenen Mitbürger.

David McGee, Parliamentary Practice in New Zealand, $3^{\text {rd }}$ Edition, Wellington 2005, S. 13.

Geddis, (oben Fn. 14), S. 101.

Ian Westbrooke/Michael Ryan, The Mathematics of Electoral District Allocation in New Zealand, Australian \& New Zealand Journal of Statistics 44 (2002), S. 267 f. 
ten Māori und den unter den Māori-Wahlkreisen registrierten Māori erzielt. ${ }^{80}$ Das Ergebnis dieser Rechnung wird im Anschluss von dem Wert der generellen Population Neuseelands abgezogen, um den Wert der generellen Wahlbevölkerung zu ermitteln. ${ }^{81}$ Diese Berechnung wird separat für die Nord und die Süd Insel vorgenommen, um hiernach die generellen Wahlkreise für die Nord Insel zu ermitteln. Der Electoral Act 1993 sieht gemäß Section 35(3)b vor, dass die Anzahl der generellen Wahlkreise für die Süd Insel auf eine Anzahl von 16 fixiert ist. ${ }^{82}$ Die sogenannte Süd Insel Quote, die sich aus der Division der generellen Wahlbevölkerung der Süd Insel durch 16 ergibt, ist damit Ausgangspunkt für die Berechnung der Anzahl der generellen Wahlkreise der Nord Insel sowie der Māori-Wahlkreise insgesamt. ${ }^{83}$ Die generelle Wahlbevölkerung der Nord Insel, sowie die Māori-Wahlbevölkerung werden hiernach durch die Süd Insel-Quote dividiert, um die Anzahl der jeweiligen Wahlkreise zu bestimmen. ${ }^{84}$

In der Folge der variablen Zahl der Māori-Wahlkreise kommt der MEO - im Gegensatz zu der Situation zwischen 1975 und 1993 - eine zentrale Bedeutung im Vorgang der Ermittlung der Anzahl der Māori-Wahlkreise zu. Sie ist maßgebliches Medium für die Erhöhung bzw. Verringerung der Māori-Wahlbevölkerung. ${ }^{85}$ Die MEO wurde erstmals 1994 unter den neuen Bedingungen abgehalten. Da die Ausübung des Optionsrechts dessen Kenntnis voraussetzt, hängt der Erfolg der Optionsphase maßgeblich von einer vorgelagerten Informationskampagne ab. Auf der Grundlage dieser Erwägungen kam es in der Folge der ersten MEO zu einem Rechtsstreit: Die Informationskampagne sei finanziell zu gering ausgestattet worden, um eine erfolgreiche Optionsphase zu ermöglichen. ${ }^{86}$ Dies führte soweit, dass einzelne Māori eine Wiederholung der MEO begehrten, da die Māori-Sitze, entgegen der hohen Erwartungen, lediglich auf eine Anzahl von fünf angestiegen waren. ${ }^{87}$ Ferner wurde beanstandet, dass die Regierung ihre sich aus dem Vertrag von Waitangi ergebende Verpflichtung zur Wahrung der politischen Interessen der Māori nicht nachgekommen sei. In Taiaroa v. Minister of Justice stellte das Revisionsgericht abschließend

80

81

82

83

84

85

86

87

Section 3(1) Electoral Act 1993.

Section 3(1) Electoral Act 1993.

Eine derartige Fixierung der Sitze der Süd Insel war erstmals durch den Electoral Amendment Act 1965 vorgenommen worden. Diese Verankerung war ursprünglich auf 25 Sitze festgesetzt und sollte der historischen Bedeutung der Süd Insel sowie der demographischen Entwicklung Rechnung tragen, dass die Bevölkerung der Nord Insel wesentlich schneller anwuchs als auf der Süd Insel. Zum Ganzen: Atkinson, (oben Fn. 1), S. 169.

Section 35(3)b Electoral Act 1993.

Sections 35(3)c und 45(3)a Electoral Act 1993.

Jonathon Boston/Stephen Levine/Elizabeth McLeay/Nigel S. Robert (Hrsg.), New Zealand Under MMP: A new Politics?, Auckland 1996, S. 71.

Geddis, (oben Fn. 18), S. 355.

Jonathan Boston/Stephen Levine/Elizabeth McLeay/Nigel S. Roberts, Courting Change: The Role of the Judiciary in Altering an Electoral System, Public Law Review 8 (1997), S. 234 f. 
fest, dass eine Wiederholung der Optionsphase nicht gewährleistet werde, jedoch eine Berücksichtigung der fehlgeschlagenen Kampagne für die nächste MEO in der Form gegeben sein müsse, dass die Regierung substantielle Mittel für den Erfolg der MEO zur Verfügung stellen müsse. ${ }^{88}$ In der Folge dieser Rechtsprechung erklärte der amtierende Justizminister, Dough Graham, 1997, dass die folgenden MEO-Kampagnen mit einer hinlänglichen Finanzierung gehalten würden. ${ }^{89}$ Durch die nachfolgenden Optionsphasen erhöhte sich die Anzahl der Māori-Sitze von anfänglich fünf in 1996, auf sechs in 1999 und sieben in 2002 und $2007 .^{90}$

\section{Problematische Aspekte der Māori Electoral Option und der Bestimmung der Anzahl der Māori-Wahlkreise}

Unabhängig von dem Aspekt der Finanzierung treten die MEO, die Volkszählung im Allgemeinen und die Māori-Sitzzuteilung im Besonderen mit weiteren problematischen Aspekten auf. ${ }^{91}$ Wie bereits beschrieben, wird die Māori-Wahlbevölkerung anhand der fünfjährlichen Volkszählung ermittelt. Innerhalb der Volkszählung besteht die Möglichkeit, jedoch nicht die Verpflichtung, Angaben über die Zugehörigkeit zu einer ethnischen Gruppe zu machen. Diese Angaben müssen der tatsächlichen biologischen Abstammung nicht entsprechen, können mithin aus Gründen eines Zugehörigkeitsgefühls zur MāoriKultur getroffen werden. Aus dieser voluntaristischen Regelung ergibt sich eine integrale Schwäche der Ermittlung der Māori-Wahlbevölkerung: die Daten könnten dahingehend verzerrt werden, dass sich ein Anteil der Māori gerade nicht dafür entscheidet, seine ethnische Zugehörigkeit, obwohl biologisch gegeben, bekanntzugeben. Weiterhin könnte sich eine Verzerrung aus dem Umstand ergeben, dass sich Personen als Māori qualifizieren, die keine tatsächliche diesbezügliche Abstammung haben. Die Verzerrung ist demnach darauf zurückführen, dass die Bestimmung der Māori-Wahlbevölkerung einzig auf der Kundgabe eines ethnischen Zugehörigkeitsgefühls basiert. ${ }^{92}$ Allerdings dürfte diese Schwäche

Eine ähnliche Regelung trifft das Gesetz über die Rechte der Sorben im Freistaat Sachsen (Sächs SorbG). So bestimmt $§ 1$ SächsSorbG: „Zum sorbischen Volk gehört, wer sich zu ihm bekennt. Das Bekenntnis ist frei. Es darf weder bestritten noch nachgeprüft werden. Aus diesem Bekenntnis dürfen keine Nachteile erwachsen." Weiterhin enthält auch die schleswig-holsteinische Verfassung in Art. 5 Abs. 1 eine korrespondierende Regelung: „Das Bekenntnis zu einer nationalen Minderheit ist frei; es entbindet nicht von den allgemeinen staatsbürgerlichen Pflichten.“ 
schlechterdings zu beheben sein, da die Bestimmung der Māori-Wahlbevölkerung innerhalb einer Gesellschaft, die seit über 170 Jahren interkulturellen Kontakt hatte, anhand biologischer Abstammungsformeln nicht nur rassistisch anmuten ${ }^{93}$, sondern überdies impraktikabel sein dürfte. ${ }^{94}$ Es bleibt jedoch fragwürdig, ob nicht eine entsprechende Verpflichtung innerhalb des Volkszählungsprozesses zur Angabe der ethnischen Abstammung eingeführt werden müsste, um genauere diesbezügliche Ergebnisse zu erzielen. Die Schwierigkeit einer solchen Obligation dürfte im Ergebnis in ihrer Durchsetzbarkeit liegen. Unabhängig von der Beeinträchtigung des Rechts auf informationelle Selbstbestimmung, das in Neuseeland freilich nicht derart ausgeprägt ist wie etwa durch Art. 2 Abs. 1 Grundgesetz in der Bundesrepublik Deutschland, ist eine Überprüfung solcher Angaben faktisch ausgeschlossen.

Die Folgen der Verzerrung der Wahlbevölkerungsdaten können sich im Ergebnis folgendermaßen auswirken: als Folge einer unterbliebenen bzw. fehlerhaften Angabe, würde jener Personenkreis, der sich trotz entsprechender Abstammung nicht als Māori gekennzeichnet hat, der generellen Wahlbevölkerung zugerechnet. Dies führt zur Unkenntnis der Behörden über eine entsprechende Verbindung des Personenkreises zur Māori-Bevölkerung. Damit werden diese Personen auch nicht über ihr MEO-Recht informiert, womit sie dieses verwirken. Im umgekehrten Fall, der Angabe der Māori-Zugehörigkeit trotz fehlender Abstammung, würde sich die Anzahl der Māori-Sitze disproportional zu den generellen Sitzen erhöhen. Besonders fragwürdig bleibt insbesondere die Bestimmung derjenigen, die keine diesbezüglichen Angaben gemacht haben. ${ }^{95}$ Diese, die integrale Schwäche bildenden Umstände sind im Ergebnis ein Grund zur Abschaffung der Māori-Sitze.

\section{Review Select Committee 2001}

2001 wurden die Regelungen hinsichtlich der Māori-Doppelwahlkreise einer erneuten Untersuchung unterworfen. Die parlamentarische Untersuchungskommission nahm sich zunächst der generellen Frage der Abschaffung der Māori-Sitze an. ${ }^{96}$ Es wurde gegen eine Abschaffung dahingehend argumentiert, dass die Māori-Wahlbevölkerung ihre Wahlkreisabgeordneten, die Verbindung zwischen Māori-Wählern und Māori-Abgeordneten, verlie-

So würde eine derartige Regelung nicht nur den deutschen Rechtswissenschaftler insbesondere an die diskriminierenden Nürnberger Gesetze, auch bekannt als Nürnberger Rassegesetze, erinnern. Zum Ganzen einführend: Bernd Rüthers, Recht als Waffe des Unrechts - Juristische Instrumente im Dienst des NS-Rassenwahns, NJW 1988, S. 2825-2835; Uwe Hansmann, Die Nürnberger Rassengesetze vom 15. September 1935 - Den Opfern zur Erinnerung, den Lebenden zur Mahnung, NJW 2005, S. 2648-2651.

Andrew Sharp, Blood, Custom, and Consent: Three Kinds of Māori Groups and the Challenges They Present to Governments, The University of Toronto Law Journal 52 (2002), S. 35. Westbrooke/Jones, (oben Fn. 91), S. 258.

96 MMP Review Committee, Report of the MMP Review Committee: Inquiry into the Review of MMP, AJHR I.23A, Wellington 2001, S. 20/21. 
ren würden. Diese Argumentation ist insoweit zutreffend, als dass eine Aufhebung der Māori-Sitze mit an Sicherheit grenzender Wahrscheinlichkeit die Aufgabe von MāoriWahlkreisabgeordneten bedeuten würde. Zwar würde sich in Folge der Abschaffung der Māori-Sitze die Anzahl der generellen Wahlkreise im Wege der Erhöhung der generellen Wahlbevölkerung, unter die dann die Māori fallen würden, ansteigen; aber es kann davon ausgegangen werden, dass unter dem Mechanismus des Mehrheitswahlrechts, welches auch unter dem System der personalisierten Verhältniswahl für den Prozess der Ermittlung der Wahlkreisabgeordneten verwendet wird, eine Auswahl von Māori-Abgeordneten wesentlich erschwert sein würde. ${ }^{97}$ Paradoxerweise war diese Schwäche des Mehrheitswahlrechts gerade die Daseinsberechtigung der gesonderten Wahlkreise, nachdem das Wahlrecht vom Eigentumserfordernis des Constitution Act 1852 gelöst worden war. ${ }^{98}$ Damit wäre die Repräsentierung der Māori maßgeblich von der proportionalen Sitzzuteilung, also der Auswahl von Listenabgeordneten abhängig. Im Falle Neuseelands ist dieser Vorgang indes von einer grundsätzlichen Unsicherheit im Umgang mit Parteilistenabgeordneten geprägt. ${ }^{99}$ Die 140 jährige, nahezu ungebrochene Tradition des relativen Mehrheitswahlrechts vor der Einführung der personalisierten Verhältniswahlsystems hat zu einer Verankerung des Gedankens geführt, dass der Wahlkreisabgeordnete, der alleinige Mandatsträger sei, der die Interessen des Wählers umfassend berücksichtigen werde, da er im direkten Kontakt zum Wähler stehe. ${ }^{100}$ Die Befürchtungen gehen dahin, dass die Parteilistenabgeordneten, die im Falle der Abschaffung der gesonderten Māori-Sitze die Repräsentation der Māori übernehmen würden, im allgemeinen tagespolitischen, durch Parteidisziplin geprägtem Geschehen, das enge Verhältnis zu den Wählern aus den Augen verlieren würden. Somit fußt die Befürchtung bezüglich der Abschaffung in weiten Teilen auf einer Unsicherheit, die sich aus dem Umgang mit dem „,neuen“ Wahlsystem ergibt. ${ }^{101}$

Darüber hinaus wurde im Bericht die Rolle der MEO untersucht. Die Bedeutung der MEO im Allgemeinen ist eng mit der Frage der Daseinsberechtigung der Māori-Sitze verknüpft, kann von dieser mithin nicht losgelöst betrachtet werden. ${ }^{102}$ Soll eine variable Māori-Wahlkreisbestimmung erhalten bleiben, muss auch eine MEO durchgeführt wer-

Ann Sullivan, The Treaty of Waitangi and Social Well-being: Justice, Representation, and Participation, in: Michael Belgrave/Merata Kawharu/David Williams (Hrsg.), Waitangi Revisited: Perspectives on the Treaty of Waitangi, Oxford, New York 2005, S. 127. McLeay, (oben Fn. 37), S. 49.

Fiona Barker/Stephen Levine, The Individual Parliamentary Member and Institutional Change: The Changing Role of the New Zealand Member of Parliament, The Journal of Legislative Studies 5 (1999), S. $105 \mathrm{f}$.

Elizabeth Mcleay/Jack Vowles, Redefining Constituency Representation: the Roles of New Zealand MPs Under MMP, Regional \& Federal Studies 17 (2007), S. 73 f.; Leigh J. Ward, 'SecondClass MPs? New Zealand's Adaption of Mixed-Member Parliamentary Representation, Political 101 Science 49 (1998), S. 125 ff.

102 Geddis, (oben Fn. 18), S. 362/363.

Comrie/Gilles/Day, (oben Fn. 89), S. 56. 
den. ${ }^{103}$ Außerdem sei die MEO ein gelungener Indikator für die Bereitschaft der Māori, die Māori-Sitze abzuschaffen. ${ }^{104}$ Diese Einschätzung scheint aber insoweit problematisch, als dass die MEO mittlerweile durch die vorgelagerte Kampagne zu einem politischen Kommunikationsprozess geworden ist, der einseitig zur Registrierung auffordert und gerade nicht umfassend über etwaige Nachteile der separaten Wahlkreise aufklärt.

Die Untersuchungskommission hob ferner hervor, dass unter dem Electoral Act 1993 ebenso wie schon unter dem Electoral Act 1956 - die Māori-Sitze nicht gesondert verfassungsrechtlich abgesichert seien. ${ }^{105}$ Einzelne Fraktionen kritisierten insbesondere, dass die Māori-Sitze durch einfache Mehrheit im Parlament abgeschafft werden könnten. Sie befürwortete, das Institut des Single Entrenchment auf die Māori-Sitze auszuweiten, um einerseits deren verfassungsrechtliche Bedeutung zu betonen und andererseits eine Änderung des status quo zu erschweren. ${ }^{106}$

Die Untersuchungskommission kam in keinem der untersuchten Kriterien in Bezug auf die Māori-Sitze zu einer konsensualen Auffassung dahingehend, Änderungen am Electoral Act 1993 vorzunehmen. ${ }^{107}$ Die Regierung entschloss sich deshalb im Ergebnis, in Ermangelung einstimmiger Empfehlungen durch das Review Select Committee, an den bisherigen Regelungen festzuhalten und damit das Wahlrecht nicht zu ändern. ${ }^{108}$

\section{Kontemporäre Diskussion}

Seit Einführung der personalisierten Verhältniswahl in Neuseeland haben Māori eine proportionalere Stellung im Parlament erlangt, als dies noch unter dem Mehrheitswahlsystem der Fall war. Weiterhin gewähren mittlerweile sämtliche Parteien mindestens einem MāoriKandidaten eine begünstigende Platzierung innerhalb der Parteiliste. ${ }^{109}$

Traditionellerweise wurden die Māori-Sitze durch Abgeordnete der Labour Partei besetzt. ${ }^{110}$ Dies änderte sich mit der Wahlsystemreform und der ersten Wahl nach Verhält-

103

104 Geddis, (oben Fn. 19), S. 361.

MMP Review Committee, (oben Fn. 96), S. 21/22.

105 Ebenso wie schon unter Section 189 Electoral Act 1956 sind nunmehr wesentliche Aspekte des Wahlrechts gemäß Section 268 Electoral Act 1993 durch das Instrument des "Single Entrenchment" abgesichert. Siehe herzu schon Fußnote 31, sowie zur Absicherung innerhalb des Electoral Act 1993: Geddis, (oben Fn. 14), S. 47; Joseph, (oben Fn. 9), S. 510 und 525.

MMP Review Committee, (oben Fn. 96), S. 24/25.

107 Stephen Church/Elizabeth McLeay, The Parliamentary Review of MMP in New Zealand, Representation 39 (2003), S. 251.

108

Select Committee, Government Response to Report of MMP Review Committee on Inquiry into the Review of MMP, AJHR A.5, Wellington 2001, S. 5.

109 Sullivan, (oben Fn. 16), S. 229.

110 Jonathan Boston, Electoral Reform in New Zealand: The Report of the Royal Commission, Electoral Studies 6 (1987), S. 107: Fiona Barker/Elizabeth McLeay, How Much Change?: An 
niswahlsystem im Jahr 1996. In der ersten Legislaturperiode unter dem neuen Wahlsystem wurden die fünf Māori-Sitze durch Vertreter der New Zealand First Party, einer von ExNational Mitglied Winston Peters geleiteten Partei, besetzt. ${ }^{111}$ In den darauffolgenden Wahlen 1999 und 2002 konnte die Tradition der Labour Party wiederhergestellt werden, so dass alle sechs bzw. sieben Sitze durch Labour gehalten wurden. ${ }^{112}$ Die Ergebnisse der Wahl zum House of Representatives 2005 brachte erneut eine Änderung dieser Situation dahingehend, dass eine neu gegründete Māori Party vier der sieben Māori-Sitze besetzen konnte. ${ }^{113}$ Dieser Umstand ist insbesondere auf einen innenpolitischen Streit über etwaige Landansprüche von Māori an Küstenstreifen im Bereich der Süd Insel zurückzuführen. ${ }^{114}$ Nachdem sich Labour und damit auch die 2002 gewählten Labour-Māori-Vertreter, aufgrund der strengen Parteidisziplin ${ }^{115}$, für den Foreshore and Seabed Act $2004^{116}$, der vorsah, dass solche Ansprüche gerade nicht durchgesetzt werden können, gestimmt hatten, formierte sich eine starke Opposition der Māori gegen dieses Stimmverhalten der MāoriAbgeordneten. ${ }^{117}$ Diese hat sich im Weiteren in der Formung der Māori Party und mittlerweile dahingehend manifestiert, dass die Māori Party im Rahmen der Parlamentswahlen 2008 fünf der sieben Māori-Sitze besetzen konnte.

Grundsätzlich lässt sich erkennen, dass Labour den Māori-Sitzen weitgehend eher befürwortend gegenübersteht, wohingegen die konservativen politischen Kräfte Neuseelands, so insbesondere National, gewöhnlich eine Abschaffung der Sitze befürwortet. National sprach sich demgemäß im Vorfeld der Wahlen 2005 für ein einheitliches Wahlrecht für alle Neuseeländer aus. ${ }^{118}$ Auf der Grundlage dieser Forderung sollten die Māori-Sitze

Analysis of the Initial Impact of Proportional Representation on the New Zealand Parliamentary 111 Party System, Party Politics, 6 (2000), S. 134.

112 Geddis, (oben Fn. 18), S. 350.

113

Die Konstellation des Split-Voting, dem Umstand, dass ein großer Anteil an Māori mit ihrer Erststimme, der Parteistimme, für Labour stimmten, brachte der Māori Party innerhalb der Parlamentswahlen 2005 und 2008 ein bzw. zwei Überhangmandate: Jack Vowles/Susan A. Banducci/ Jeffrey A. Karp, Forecasting and Evaluating the Consequences of Electoral Change in New Zealand, Acta Politica 41 (2006), S. 272/273.

114 Sarah Maddison, Ideas from 'across the ditch'? Wedge politics in the 2005 New Zealand Election, Australian Journal of Political Science 41 (2006), S.. 430; Andrew Geddis, The General Election in New Zealand, September 2005, Electoral Studies 25 (2006), S. 811.

Im Gegensatz zur Bundesrepublik Deutschland besteht in Neuseeland ein sogenanntes Parteimandat, dass eine strenge Parteidisziplin erlaubt. Zum Ganzen: Andrew Geddis, Proportional Representation, 'Party Hopping' and the Limits of Electoral Regulation: A Cautionary Tale from New Zealand, Common Law World Review 35 (2006), S. 24.

116 Paul McHugh, Setting the Statutory Compass: The Foreshore and Seabed Act 2004, New Zealand Journal of Public and International Law 3 (2005), S. 255-283.

117 Jack Vowles, New Zealand, European Journal of Political Research 45 (2006), S. 1217.

118

Bill English, The Treaty of Waitangi and New Zealand Citizenship, The New Zealand Law Journal (2002), S. 258. 
abgeschafft werden. Nachdem National über keine alleinige Mehrheit im Parlament verfügte, wurde die Abschaffung der Sitze im Rahmen der Koalitionsverhandlungen mit der Māori Party, die als einziger Koalitionspartner zur Verfügung stand, jedoch zu einer disponiblen Causa. ${ }^{119}$ Auch nach der Amtsübernahme Nationals 2008 konnte sich die Māori Party in diesem Punkt durchsetzen, wenngleich die Abschaffung der Māori-Sitze innerhalb des Wahlkampfes nur eine untergeordnete Rolle spielte. ${ }^{120}$

\section{Bewertung und Ausblick}

Regelungen, die die Repräsentation von Minderheiten garantieren, stellen in wahlsystematischer Hinsicht keine Besonderheit dar. ${ }^{121}$ Die Außergewöhnlichkeit der Māori-Sitze liegt vielmehr in ihrer 140 jährigen Tradition. ${ }^{122}$ Die garantierten vier Māori-Sitze brachten eingangs - wenn auch nur disproportional - zumindest ein Mindestmaß an Repräsentation. Die Daseinsberechtigung der separaten Wahlkreise mit ihren garantierten Sitzen hat sich mit der Zeit dahingehend gewandelt, dass sie anfänglich zur Durchbrechung des faktischen Ausschlusses der Māori vom Wahlrecht dienten und später die Schwächen des Mehrheitswahlrechts im Hinblick auf Minderheitenrepräsentierung ausgleichen sollten. Unter dem nunmehr geltenden personalisierten Verhältniswahlsystem ist eine derartige Daseinsberechtigung insofern fraglich, als dass das geltende Wahlsystem, wie dargelegt, grundsätzlich adäquaten Minderheitenschutz gewährleistet. Dementsprechend argumentiert $O^{\prime}$ Connor, dass der Sinn des Māori Representation Act 1867 längst ausgelaufen sei. ${ }^{123}$

Diese technischen Argumente verkennen indes die sozio-kulturelle Rolle der Māori innerhalb der Gesellschaftsstruktur Neuseelands. ${ }^{124}$ Nach einer langen Phase der Negierung, kam es insbesondere Mitte der 1970er Jahre zu einem Prozess der Anerkennung der Rechte der Ureinwohner. Die Royal Commission on the Electoral System sprach in ihrer Untersuchung im Zusammenhang mit den Māori-Sitzen von deren symbolischer Signifikanz. ${ }^{125}$ Andrew Geddis beschreibt die Sitze als „repräsentatives Sicherheitsnetz“. Nach

119

120 Grant Fleming, John Key announces he has numbers to govern, New Zealand Herald, 16. November 2008 .

121

Pippa Norris, Electoral Engineering: Voting Rules and Political Behavior, New York 2004, S. 209 ff.; Andrew Reynolds/Ben Reilly/Andrew Ellis, Electoral System Design: The New International IDEA Handbook, Stockholm 2005, S. 122 ff.

122 Die ersten Wahlen nach dem Māori Representation 1867 wurden 1868 durchgeführt: Sullivan, (oben Fn. 16), S. 219.

123 R.J. O'Connor, The Future of Māori Representation in Parliament, New Zealand Law Journal (1991), S. 177.

124 John H. Wallace, Reflections on Constitutional and Other Issues Concerning Our Electoral System: the Past and the Future, Victoria University of Wellington Law Review 33 (2002), S. 311.

Royal Commission on the Electoral System, (oben Fn. 42), S. 85. 
ihm seien die Māori-Sitze charakteristischer Ausdruck eines Bedürfnisses nach Anerkennung der besonderen Rolle der Māori als indigenes Volk Neuseelands. ${ }^{126}$

Insofern verkennt eine sich einzig an der Logik der Argumentation orientierende Auseinandersetzung die tief verwurzelte Befürchtung der indigenen Bevölkerung Neuseelands, bei der politischen Willensbildung übergangen zu werden. Zusätzlich spielt in diesem Zusammenhang die Besorgnis eine Rolle, dass die Abschaffung der Māori-Sitze bei einer anschließenden Rückkehr zum Mehrheitswahlrecht, als Folge eines fehlgeschlagenen Versuchs der Erprobung des Verhältniswahlrechts, erneut zu einem wahlrechtlichen Ausschluss der Māori führen könnte. Ein Ausweg aus dieser Unsicherheit könnte sich mit fortschreitender Zeit aus einer gewonnenen Sicherheit im Umgang mit dem System der personalisierten Verhältniswahl ergeben. Der Einschätzung des ehemaligen Vorsitzenden der Royal Commission on the Electoral System, John Wallace, zufolge befänden sich die Māori derzeit in einem Prozess der Anerkennung des Wertes der personalisierten Verhältniswahl. $^{127}$

Sollten diese politischen Hürden genommen sein, wäre eine Anlehnung an die deutsche Regelung zum Schutz der Repräsentation von nationalen Minderheiten wünschenswert wie bereits 1986 von der Royal Commission empfohlen. § 6 Abs. 6 Satz 2 Bundeswahlgesetz sieht eine Ausnahme für die $5 \%$-Hürde für Parteien nationaler Minderheiten vor. ${ }^{128}$ Mai Chen kritisiert in diesem Bezug die Schwierigkeit der Bestimmung von Parteien, die sich ausschließlich auf Māori-Interessen konzentrieren. ${ }^{129}$ Zwar tritt auch im deutschen Fall die Definition der Partei einer nationalen Minderheit mit Schwierigkeiten auf; ${ }^{130}$ aber hat insbesondere das schleswig-holsteinische Beispiel der Minderheitenrepräsentation gezeigt, dass eine erfolgreiche Gewährleistung der Repräsentation im System der personalisierten Verhältniswahl erreichbar ist.

\section{Zusammenfassung}

Neuseeland ist besser mit der Abschaffung der Māori-Sitze beraten. Das System der personalisierten Verhältniswahl eröffnet hinlängliche Möglichkeiten für eine zufriedenstellende Repräsentation der Māori. Überdies sollte im Fall der Abschaffung der Sitze eine Regelung entsprechend des $\S 6$ Abs. 6 S. 2 Bundeswahlgesetz bzw. $\S 3$ Abs. 1 S. 2 Wahlgesetz für den Landtag von Schleswig-Holstein übernommen werden, um eine Erleichterung der

127 - Wallace, (oben Fn. 124), S. 312.

127 Wallace, (oben Fn. 124), S. 312.

Angelika Kühn, Privilegierung nationaler Minderheiten im Wahlrecht der Bundesrepublik Deutschland und Schleswig-Holsteins, Frankfurt am Main, Bern, New York, Paris 1991, S. 6.

Mai Chen, Remedying New Zealand's Constitution in Crisis: Is MMP part of the Answer?, New Zealand Law Journal (1993), S. 28.

Wolfgang Schreiber, Handbuch des Wahlrechts zum Deutschen Bundestag: Kommentar zum Bundeswahlgesetz, 7. Auflage, Köln, Berlin, Bonn, München 2002, § 6 Rn 22. 
proportionalen Sitzzuteilung für Māori Parteien zu ermöglichen. Das deutsche Modell der Erhöhung der Chancen des Zugangs von Parteien nationaler Minderheiten hat insbesondere im Falle des Südschleswigschen Wählerverbandes verlässliche Dienste geleistet und dürfte deshalb als konstruktives Vorbild im Falle der Abschaffung der separaten Wahlkreise dienen.

Der Prozess der Abolition der dualen Repräsentation ist im Ergebnis ein Prozess der politischen Konsensfindung. Insoweit dürfte eine Abschaffung der Sitze erst mit einer Sicherheit im Umgang mit dem personalisierten Verhältniswahlsystem, sowie der Erkenntnis der Stigmatisierung der Māori-Wahlkreise durch die Māori in erreichbare Nähe rücken. Wie bereits Hans Meyer zutreffend artikuliert hat, sind Parlamentarier in keinem anderen Bereich der Gesetzgebung derart befangen wie im Bereich des Wahlrechts. ${ }^{131}$ Dieser Grundsatz gilt auch und besonders im Falle der Auseinandersetzung mit den Māori-Sitzen für Neuseeland. Handbuch des Staatsrechts der Bundesrepublik Deutschland, Band II, Heidelberg 1987, § 37 Rn 37. 